\title{
Which Sung Pitch Range is Best for Boys during Voice Change?
}

\author{
Authors \\ Dr Jenevora Williams PhD \\ Evolving Voice, Guildford UK \\ ORCID 0000-0001-7662-6702 \\ jw@jenevorawilliams.com \\ Professor Graham F Welch PhD \\ Established Chair of Music Education, University College London UK \\ ORCID 0000-0003-2258-8039 \\ graham.welch@ucl.ac.uk \\ Professor David M Howard PhD \\ Established Chair in Electronic Engineering, Royal Holloway, University of London UK \\ ORCID 0000-0001-9516-9551 \\ david.howard@rhul.ac.uk
}

\begin{abstract}
The question of how and what boys should sing during adolescent voice change has challenged educators and choir leaders for the last century. As the larynx enlarges during adolescence, most boys will follow the descending pitch of their speaking voice and move to singing vocal parts with a lower pitch range. Occasionally a boy may continue to sing in his treble (soprano) range while his larynx is growing and his speaking pitch is lowering. There is much opinion on the wisdom of such practices, but until now there has been no quantifiable evidence to illustrate the discussion. The established historic and cultural practices can now be reassessed in the light of quantitative analyses of vocal function. This longitudinal case study used electroglottographic measures from one boy over a three-year period to investigate the efficiency of vocal fold adductory behaviour in both pre-change and mid-change singing. In the first recording he is aged 10 years old and has an unchanged voice. In the second recording he is aged 13 years old and has a speaking voice in the Cooksey Stage III of voice change. Up to and including the time of the second recording, he had chosen to remain singing exclusively in his treble (soprano) range. The comparison between the two recordings of the observed regularity and efficiency of vocal fold adduction suggests that singing in this pitch range has become less healthy and effective; this is also represented in the overall perceived vocal comfort levels heard in the recordings. The implications for educators are that the longitudinal development of singing habits will be enhanced for boys who move to singing with their new baritone range as their larynx grows, rather than remaining in a treble range.
\end{abstract}

\section{Keywords}

boy, voice change, adolescent, pitch range, chorister 
Which Sung Pitch Range is Best for Boys during Voice Change?

\section{Introduction:}

The ways in which boys engage with singing varies across different cultures and traditions; this paper will address those based on the Anglo-Catholic classical choral model. In the Cathedral choral tradition, boys have traditionally remained singing the treble part (soprano) until their adolescent laryngeal growth renders this either impractical or uncomfortable, or until they reach the upper age limit for the choir. In the twentieth century, there were several movements [1-3] to suggest that boys could remain singing throughout puberty, but that their voice would develop more healthy phonatory habits in the longer term if they were encouraged to move down through the choral parts of alto, tenor or bass, alongside the lowering pitch of their speaking voice. In order to allocate the most appropriate vocal range, the boy's speaking voice needs to be assessed frequently during his period of growth; this is because of the close correspondence between spoken pitch and sung tessitura [4]. Chronological age is only a guide for biological age; there will always be outliers at either end of the development distribution curve who will appear to be outside the median developmental stage [5]. At worst, the decisions concerning the allocation of choral parts are based on chronological rather than biological age; for example, in some school choirs all boys up to the age of 13 will sing treble, 13-14 alto and 14-16 tenor or bass. This fits the average vocal pitch ranges of each age group and it removes the need for individual assessment, but risks inappropriate labelling; many boys in this system will simply be unable to sing their allocated voice part due to their developmental mismatch with the average for their age. In many of the Cathedral foundations, the decision to remove a boy from the treble part will be based on perceived comfort levels, rather than his physical developmental stage; herein it is proposed that this method of evaluation is too subjective and physiologically inappropriate to be reliable.

In terms of research in this area, the principal investigator was initially John Cooksey, who assessed and monitored hundreds of boys' voices from the ages of 11 to 16 years [1]. He noted five distinct Stages of voice change, measurable by assessing the average pitch of the boy's speaking voice (Figure 1). These five Stages, assessed by listening, were subsequently compared alongside Tanner's five stages of physical 
development during adolescence, which are assessed by physical examination [6]. The physical and vocal stages of development were shown to occur simultaneously [7], and these are both known to be a direct consequence of increasing testosterone levels in the body [8]. As a result, we are confident in making a reasonably accurate judgement of the boy's overall adolescent growth stage simply by listening to his speaking voice.

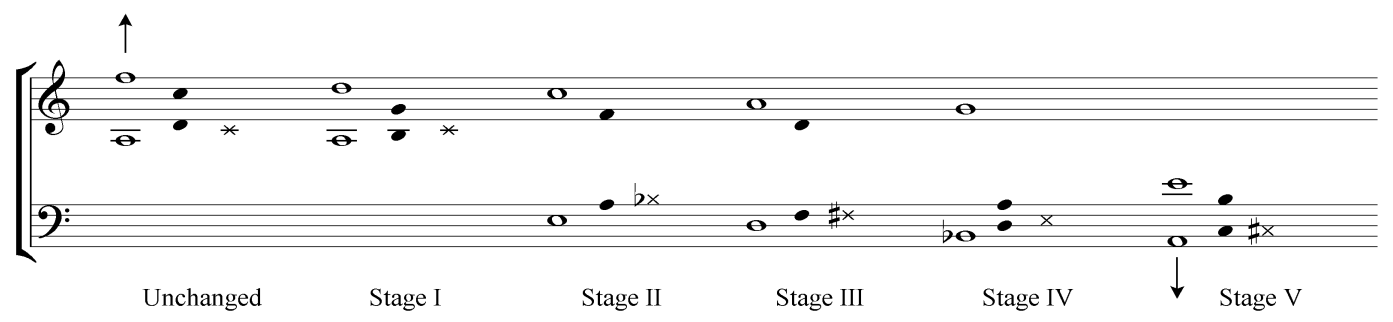

Figure 1 - The pitch ranges of each Stage of male voice change [9]

(Extended singing range (unfilled note), overall speech range (filled note), speech fundamental frequency (cross).)

This average fundamental frequency of the speaking voice can be assessed using various software apps (such as OperaVOX); if these are not available, a rough measure (to the nearest $+/$ - one semitone) can be ascertained simply by asking the boy to count backwards from 20 and noting the pitch at which his voice settles most comfortably, this most reliable portion of the exercise will be between the numbers 13 and 9. This pitch is usually three to four semitones above his lowest comfortable singing pitch [10]. If the boy is singing outside his most comfortable pitch range, there may be signs of vocal discomfort or strain which might be visual (increased jaw or neck tension, changes in posture) and/or aural (voice becoming hoarse, scratchy, breathy or constricted) [11]. These subjective criteria will become increasingly evident beyond pitches that are towards the extremes of the singing range. There tends to be more stability and less individual variation around the lower pitch range limit throughout the different stages of voice maturation than around the upper pitch range limit [12]. Therefore, it is more reliable to judge the developmental Stage by the lower singing range and by the average fundamental frequency of speech.

Judging and labelling the developmental stage is integral to an ongoing debate between at least two schools of thought regarding the stages or phases of voice 
change for males. The first, or "limited range" school believes that boys' voices change predictably, lowering gradually according to a rather prescribed pattern. Irvin Cooper was the originator of this theory (the 'Cambiata' concept)[13]; two of his students continued to advocate this approach: Don Collins (founder of Cambiata Press) and John Cooksey (the 'Eclectic' theory) [1]. Cooksey expanded upon Cooper's work and included five Stages for the male pubertal voice during change (see Figure 1). The second, or "extended range" school believes that boys' voices can change slowly or quickly and may not be limited to a mid-voice comfort range of an octave or less during puberty. Frederick Swanson was the originator of this school and contemporary advocates have included Henry Leck [2] and Kenneth Phillips [3]. It has been suggested that the former method of vocal assessment (the 'Cambiata' concept) may be more appropriate in an average school setting, as it is relatively easy to apply. The more individual, extended range school, allowing boys to sing in their entire available range, may be more suited to the highly skilled boy singers in the Cathedral choirs [14]. However, successful application of this method of assessment also relies on the skill and knowledge of the teacher concerned.

\section{Adolescent Growth}

The duration of male puberty can be from eight months to over four years [15], commencing at any time between the ages of ten and fifteen; the average age of onset is 12.5 years [16]. Physical growth through adolescence is in growth spurts or stages. The growth of the larynx commonly mirrors the observable overall growth of the individual [17]. If the individual is undergoing a growth spurt with a noticeable height gain, the larynx and its associated skeletal and muscular anatomy will be undergoing comparable enlargement. This is then followed by a period of stabilization. This growth pattern is caused by changes in hormonal levels and cannot be accelerated or decelerated except by artificial hormonal input [18]. Although the habitual pitch of the speaking voice is mainly dependent on vocal fold length; the noticeable drop in pitch observed between Stages 3 and 4 is largely due to increased vocal fold mass more than sudden elongation of the vocal folds [19]. Skeletal growth and muscle mass are directly related [20]; an increase in skeletal size will usually result in increased muscle length. This increased muscle length precedes increased strength, temporarily affecting overall co-ordination. This mismatch in size and physical co-ordination is observable immediately following an overall growth spurt. The boy will not only have 
a skewed proprioceptive awareness of his body in relation to the world around him, he will also have reduced muscular co-ordination [21]. During this time, all his previously acquired motor skills will be compromised until his muscular strength catches up with the increase in muscular length. From this knowledge, we could infer that a boy whose larynx is growing rapidly may be less able to co-ordinate the fine adjustments necessary for skilled vocal behavior. This could be an explanation for the reduced vocal pitch-range in mid-puberty as illustrated in Figure 1. It is also possible that this reduced coordination will result in measurable and audible instabilities in the voice, such as huskiness.

\section{Measuring vocal efficiency and healthy function}

The sheer number of variables to consider serves to confound any evaluation of best practice in singing: efficient muscular function, economic effort levels, and effective musical expression. Even attempts to pinpoint the most appropriate pitch range will bring associated caveats and subjectivity. Any singer will inevitably spend some of their time working at the extremes of vocal ability because it is often where the most exciting and emotionally driven vocalizing occurs [22]. The degree of balance between these extremes and 'comfort' will vary across individuals, depending partly on vocal technique and skill, but also on the specific nature and particular robustness of their changing vocal system. Perceived levels of comfort have been used to evaluate technical efficiency across genres in singers [23]; however, this may not be appropriate when measuring subtle changes in comfort levels over time. Growth changes over time are not necessarily noticeable, as represented by the 'boiling frog syndrome' (It is said that if one drops a frog into a pot of boiling water, it will jump out. But if one places a frog in a pot of cool water and gradually increases the temperature, the frog will remain in the water until it is boiled). The boy will not necessarily be aware of the subtle alterations in phonatory efficiency within a particular pitch range if he is using that range on a daily basis.

In all physical activity, efficient usage is essential for injury prevention [24] and to get the best from the biological structures. In singing, a decrease in efficiency will be linked to a compromised level of healthiness, whether or not the boy concerned perceives this. If taken further, a decrease in efficiency will incrementally increase the stress on the tissues that could eventually lead to a collapse of the system. Physical 
stress theory [25] states that a physical activity should function within the maintenance stress range of the individual, and that this depends on prior strength and training. An increase in levels of stress to the tissues, depending on the magnitude, time and direction of movement, will ultimately cause the system to reach the maximum stress threshold. This process, although requiring a gradual change over time, will not necessarily be noticed. As the maximum stress threshold is reached, the ability of the system to perform the function will exhibit an acute and often uncontrollable response, the system will collapse with either a drastically reduced ability to function, or in some cases, acute pain. This appears to be a single event, and the singer will seek to identify it as such. It is, however, the result of a longer-term cumulative build-up of stress to the system.

This tipping point into system collapse fits with Catastrophe theory [26], in which cognitive anxiety is a factor alongside physiological arousal and performance outcomes. This is illustrated in Figure 2.

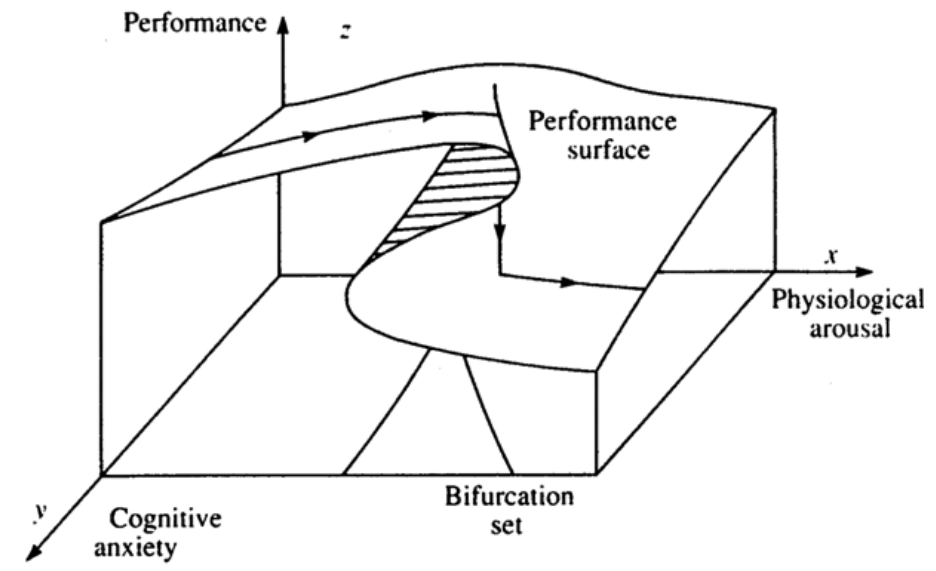

Figure 2: Catastrophe model of performance [26]

The inverted U from Yerkes-Dodson Law [27] relies on two variables: arousal and performance. As arousal increases so does performance, until a peak is reached whereupon any further increase in arousal will result in a decreasing level of performance. The addition of a third variable, that of an increase in cognitive anxiety, can lead to a significant bifurcation of the system, leading to a catastrophic change in performance outcome. This would suggest that collapse or injury is more likely to occur during the increased cognitive anxiety of a performance rather than during 
rehearsal, given that music performance anxiety (MPA) is highest at the beginning of a performance [28]. Cumulative trauma disorders from misuse or overuse will have a chronic build-up with a tipping point into acute symptoms [29]. The same can happen in the healthy function of the voice; performers who have experienced sudden voice loss will seek for 'in the moment' causality, whereas some functional voice loss is an acute response to a chronic disorder [30]. In boys experiencing adolescent voice change who continue to sing in the treble pitch range, this acute response can manifest as a sudden inability to sing as before; hence the term voice-break. There are many documented experiences of overnight voice-break; yet we know that this extent of growth is not possible overnight: the experience must in fact be the tipping point of collapse as a result of reaching the maximum stress threshold.

The teenage boy will be experiencing this rapid level of growth for the first time. He will not necessarily know that the subtle changes in his vocal ability have the potential to cause a stress-related injury. His teachers and musical directors have a direct duty of care to advise him of the consequences of this and to prevent it from happening by encouraging him to sing in his lower pitch range at the appropriate stage of his vocal development.

If comfort levels and extended pitch range may not be appropriate measures of vocal efficiency, given their subjective nature; an effective measure could be an objective assessment of the vocal fold adductory behaviour. Efficiency of vocal fold adduction can be measured from the Phonation Threshold Pressure, the rate of vocal fold closure, or the relationship between the percentage of glottal closure in each vibratory cycle and the acoustic output [31]. Efficient vocal fold adduction will give the highest acoustic output for the least adductory effort [32].

\section{Methodology}

This research was part of a larger study looking into the vocal health and development of boy choristers in the UK [12]. These longitudinal observations were made as some of the senior choristers began their adolescent voice change. In this part of the study, one boy (boy C) was recorded at the age of 10 years 9 months, when his voice was unchanged. He was subsequently recorded at the age of 13 years 7 months, when he was in Cooksey's Stage III of voice change (see figure1). For the duration of this 
time, the boy had remained singing the treble (soprano) part in the choir. Most of the boys beyond Stage II had moved away from singing the upper voice parts. However, this particular boy had chosen to remain singing as a soprano; he self-reported as being comfortable doing so and he was determined to continue. As such, he was an unusual and unique example in this particular cohort of singers, hence the focus for this case study on one boy participant.

The recordings took place in one of the school classrooms that was well known to the choristers and relatively isolated acoustically from the rest of the school. Recording sessions were at the end of the school day during the boys' private study time. The participants followed a specially designed vocal behaviour assessment protocol that embraced speaking and singing activities. These included the reading aloud of an established spoken age-appropriate text ('Arthur the Rat') - commonly used in voice clinics, vocal exercises (scales, pitch glides and messa di voce), plus the singing of the first verse of a hymn tune that had been identified as being well-known to the choristers. The resultant vocal behaviour was recorded digitally prior to (a) perceptual and (b) acoustic analyses. A Senheisser MKH-20 omnidirectional Microphone was fixed at a constant distance $(30 \mathrm{~cm})$ from the mouth by means of a head-mounted boom (see Figure 3). Initially, the boy was asked to sustain a [a] vowel and the level was measured in dBSPL (A-weighted), using a SPL meter (Radioshack) with its microphone placed alongside the Senheisser MKH-20 microphone. This was used to calibrate the levels of the recordings (the gain control was then not altered throughout the recording). Field notes were also taken by the lead author at each recording session. The recordings were made in a standard school classroom. The boy was placed in the centre of the room. The music stand was angled such that the first reflection did not reach the microphone. The long-term average spectrum (LTAS) of selected recordings was analysed (analysis bandwidth $300 \mathrm{~Hz}$, range up to $10 \mathrm{kHz}$ ). The graph was obtained using the program 'Wavesurfer' [33]. The electrolaryngograph signal gives a waveform showing the time-varying change of vocal fold contact area with time. This was observed in the program 'Wasp' [34] and the image captured using 'Snagit' [35].

Voice source data were collected in parallel to the acoustic data via an electrolaryngograph (Lx) for subsequent analysis. The Lx data represent changes in 
vocal fold contact area, which gives a basis for assessing the activity of the opening/closing mechanism of the vocal folds in running speech or singing. Subsequently, selected portions of the Lx signal were inspected in order to aid the investigation of perceptually salient vocal events in the data to see if there was any independent voice source evidence of variations in vocal fold contact waveforms that might explain variations in the perceptual data.

Contact quotient (known as CQ) is a measurement obtained from the electrolaryngograph [31]. The Lx measure produces a waveform which can give a measure of the degree and duration of vocal fold contact in each cycle [36]. This can be interpreted, in general, as a measure of vocal efficiency. Note that the electrolaryngograph (Lx or ELG) waveform is plotted with vocal fold contact area increasing vertically upwards [37]. A faster closing rate of the vocal folds caused by the folds snapping together more quickly or with more force (indicated by a steeper rising slope on the Lx waveform corresponding to a more rapid change in vocal fold contact area) will result in a louder perceived sound [38]. If the contact phase is too long, it can be an indicator of pressed phonation [39]; this can occur as a result of vocal strain, and will add to the overall vocal loading and the potential for fatigue [40].

By observing the resultant long-term average spectrum (LTAS), it is possible to quantify the variation in fundamental frequency, the distribution of the upper harmonics, and the overall intensity of the signal. It is important to calibrate the sound pressure level (SPL) of each recording. 


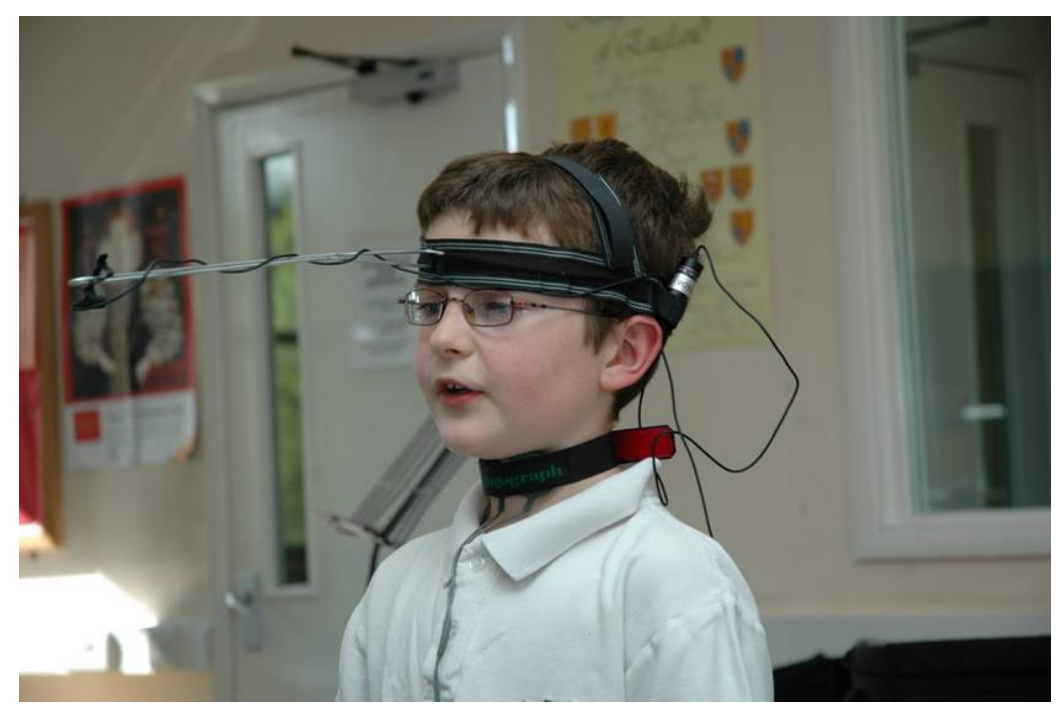

Figure 3: Boy with electrolaryngograph and microphone, an illustrative example of the recording set-up (reproduced with permission)

The research protocol was accepted under the British Education Research Association [41] code of ethics; all adults involved had up-to-date enhanced UK Criminal Records Bureau (now called DBS) clearance to work with children. The recording sessions were conducted in a room within the school. The door was kept closed in order to minimise external noise ingress. The boys had the electrolaryngograph and microphone equipment physically fitted by an adult (see Figure 3). At the choir school there were three to four adults in the room for each session when there were between one and four boys present. All rooms used had a clear glass panel in the door so that the proceedings could be observed by a third party from outside the room at any time. The researcher (and, on one occasion, with the then supervisor) met with key management personnel, including the Headmaster of the school, the Director of Music in the school, the Director of Music in the cathedral, and a senior cathedral authority. Once their agreement for the research had been secured in principle, each chorister and his parents were approached in writing to seek their agreement to participate.

\section{Results}

The Lx waveform gives information on the degree of vocal fold contact. This can be used to assess the efficiency of phonation and can show the changes in vocal fold contact patterns within and across pitches with the onset of adolescent voice change. 
The perceptual quality of the upper pitches of boy $\mathrm{C}$ at Stage 111 of voice change is of an accomplished treble voice. It is only when this is compared directly with the recording of the boy pre-puberty that a difference in comfort level becomes more obvious. In the second recording he has higher levels of perceived strain and tongue root pressure. It is only by looking further into the data, and comparing the longitudinal results, that evidence of inefficiency can be observed.

Figures 4 and 5 show one cycle of the Lx waveform of a c5 (approx. 520Hz) on the word 'sweet', taken from the recording of the hymn tune. The initial observation is that in the later recording, the cycle has a longer contact phase as indicated by the larger percentage of each vibratory cycle being occupied by the positive peak. This would be expected in louder voicing; it is also observed in pressed phonation [42]. The CQ in Fig 5 (average $=38.34 \%$ ) is not long enough to be injurious, but it is longer that the CQ in Fig 4 (average $=24.78 \%$ ) [31]. The output sound levels in both recordings for this sample were similar. Therefore the inference is that the boy's voice is working harder on the second occasion in order to produce a similar level of sound as in the earlier recording.

Boy $\mathrm{C}$ aged $10 \mathrm{y} 9 \mathrm{~m}$ - singing $\mathrm{C} 5$ on 'sweet'

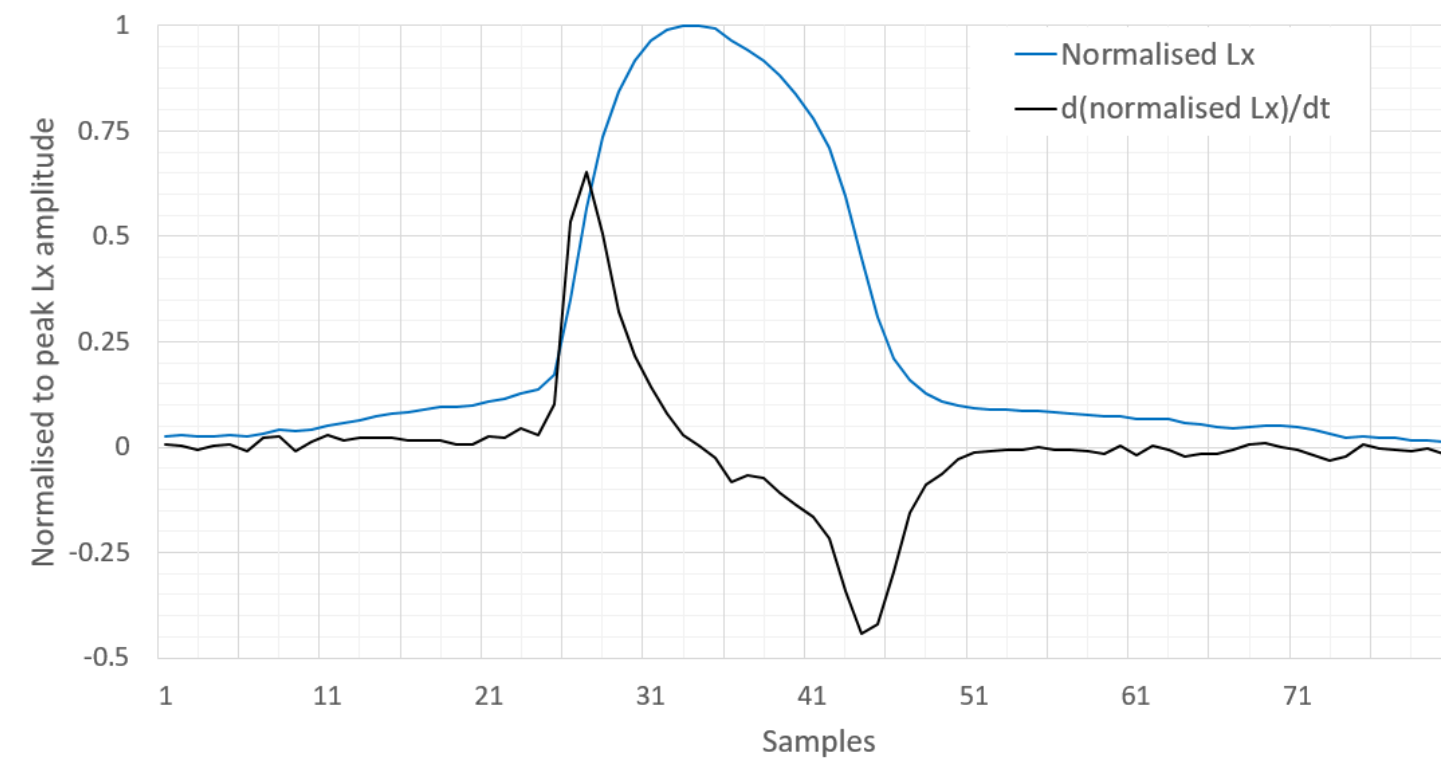

Figure 4: One normalised cycle of EGG waveform (blue) and its time differential [43] of vocal fold excitation in boy $\mathrm{C}$, aged 10 years 9 months, singing the note $\mathrm{C} 5$ $(520 \mathrm{~Hz})$ on 'sweet'. 


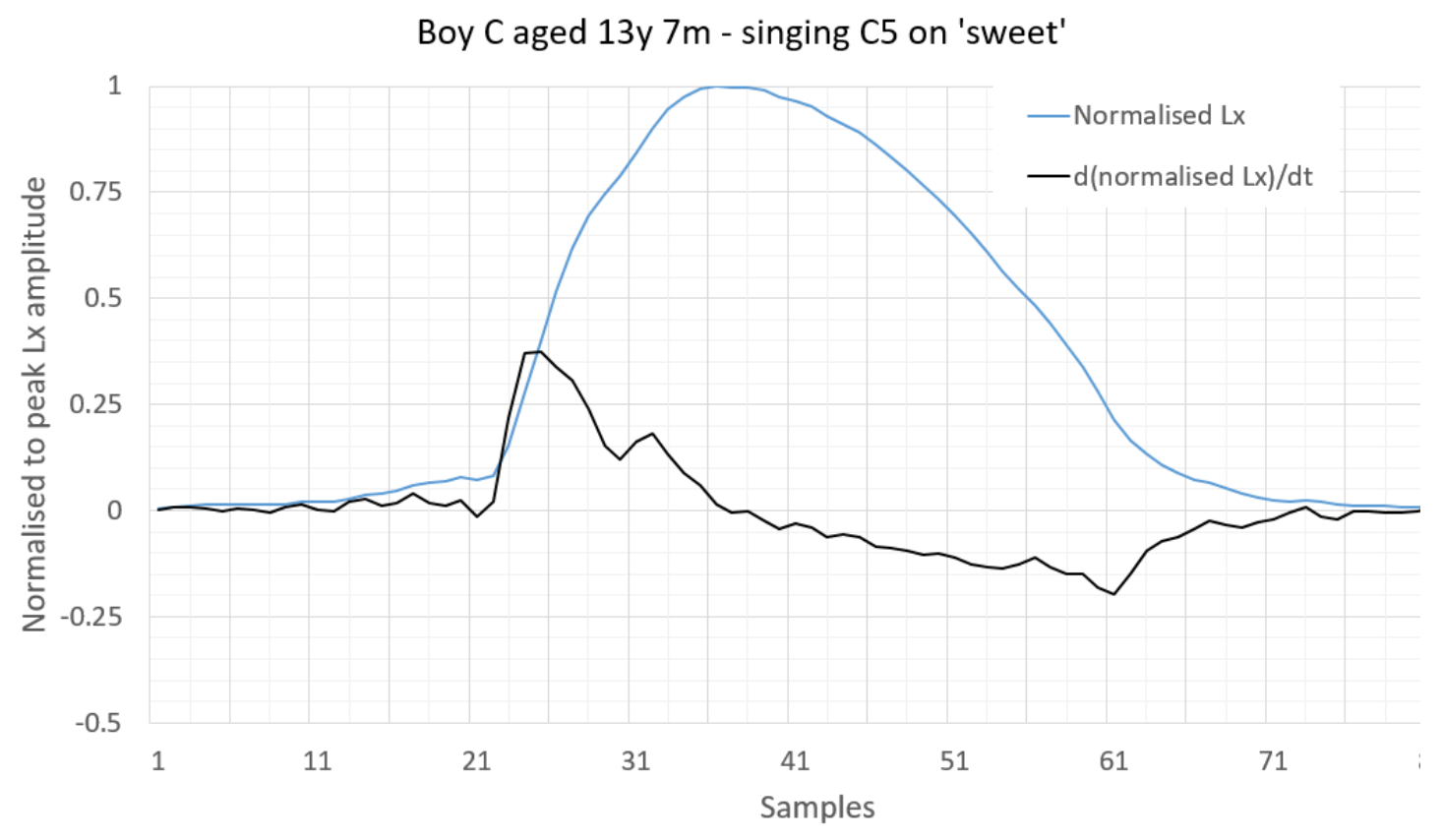

Figure 5: One normalised cycle of EGG waveform (blue) and its time differential [43] of vocal fold excitation in boy $\mathrm{C}$, aged 13 years 7 months, singing the note $\mathrm{C} 5$ $(520 \mathrm{~Hz})$ on 'sweet'.

The superimposed line in black (smaller waveform) on Figures 4 and 5 is the first derivative, which shows the steepness of the rising and falling parts of the Lx curve; this indicates the rate of closing and opening of the vocal folds respectively. The earlier recording, Figure 4, has a more rapid change in vocal fold contact area at closing and opening. This not only produces a stronger excitation impulse but also boosts the higher frequency spectral component in the region of 5,000 $\mathrm{Hz}$ [44]. This can be seen in the LTAS plots in Figures 6 and 7. The earlier recording shown in Figure 6, when boy $\mathrm{C}$ had an unchanged voice, showed a more efficient pattern of vocal fold contact; the boost to the upper frequencies can be seen circled in red. This frequency boost is heard as a clearer, more ringing tone [45] and as it is taking advantage of more efficient vocal fold vibration rather than muscular effort, it is likely to be reducing the risk of overall vocal fatigue. It is worthy of note that there appears to be less evidence of a second formant in the later recording. Perhaps this is due to the boy adapting his resonance strategies to a lengthening vocal tract. 


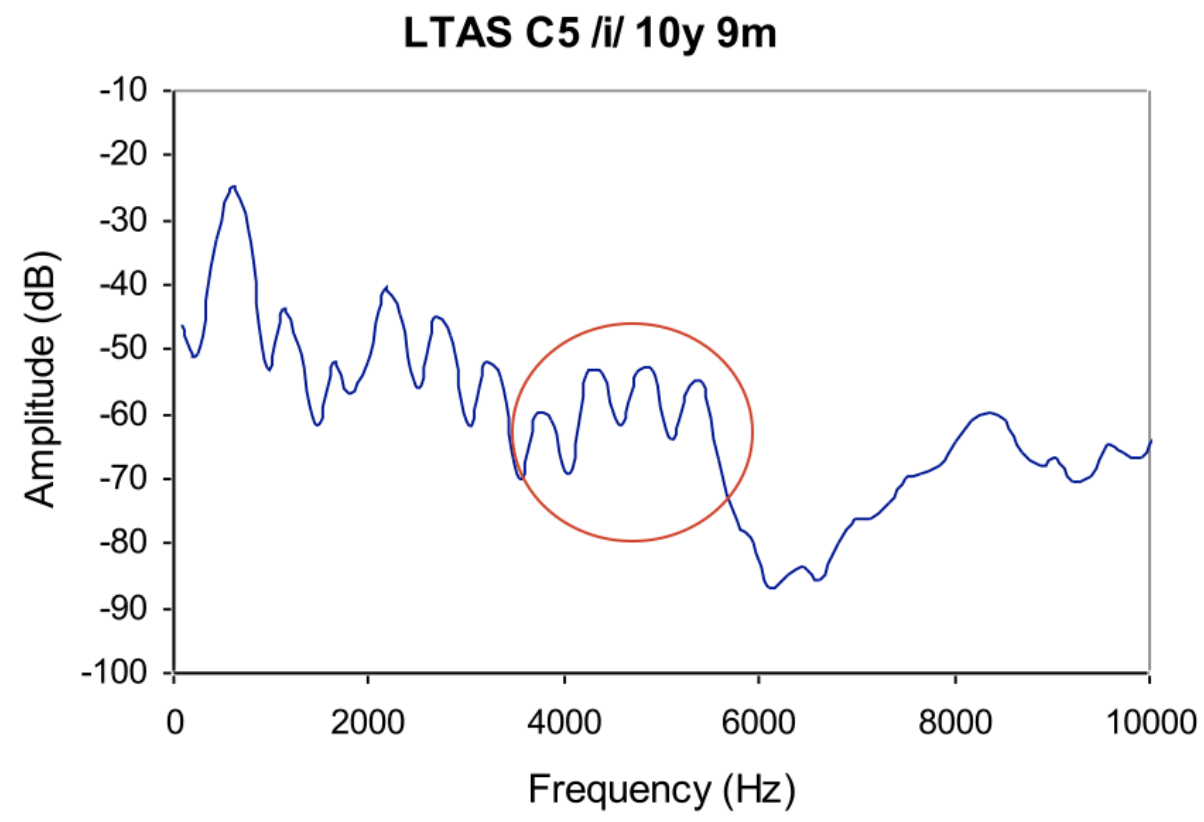

Figure 6: LTAS of [i] vowel on c5 $(520 \mathrm{~Hz})$, Boy C aged 10 years 9 months

LTAS C5 /i/ 13y $10 \mathrm{~m}$

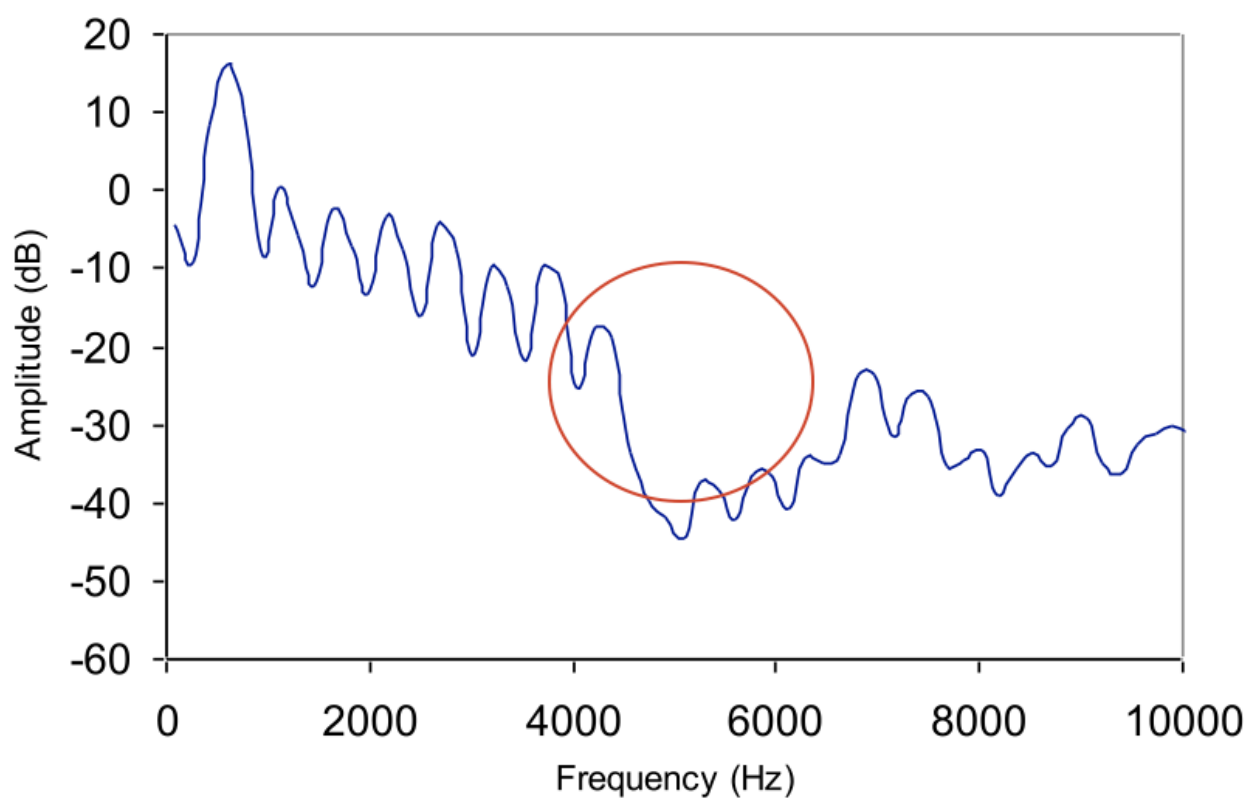

Figure 7: LTAS of [i] vowel on c5 $(520 \mathrm{~Hz})$, Boy C aged 13 years 10 months

This significant observation is one form of quantifiable evidence to show a difference in vocal behaviour between the unchanged voice singing in the treble range and Stage 111 of voice change at the same sung pitch. If the intention is to measure CQ as a single 
variable, an extended closed phase gives a more efficient acoustic output due to the time reduction in sub-glottal damping [31]. In this instance, the sound amplitude remains similar, despite the higher CQ in the second recording. In addition, there is a loss of upper partials due in part to the slower rate of vocal fold closure [46]. These data analyses suggest that the adductory behaviour of the vocal folds during voice change is less efficient than that of the younger boy. Less efficient vocal fold activity, or increased effort for a decreased acoustic output, leaves the singer more prone to fatigue or injury.

\section{Discussion}

It is possible that, as the larynx grows, a boy can retain the ability to sing with a treble (soprano) sound quality and range. The question to consider is whether this extended use of the upper pitch range is advisable during adolescent voice change and, if so, for how long. This can now be viewed in the light of evidence relating to the reduced efficiency of voice production, despite the appearance of comfortable, healthy phonation; and secondly, the potential for sudden onset of voice loss as a result of accumulated stress.

These findings could inform the practice of singing teachers and choir trainers. The current advice to boys during voice change tends to be to sing in the lowest comfortable pitch range for the duration of this time. These results may suggest that it is possible to maintain a singing voice with a high tessitura, at least during some of the early Stages of voice change, but at some point a judgment call will need to be made on the potential for injury. The Cooksey system of five Stages may be a useful guide for the majority of teachers and singers. It is relatively simple to implement, and it is unlikely to be misinterpreted. The 'extended range' school of thought may be more applicable to experienced boy singers, who may have a larger accessible pitch range. Nevertheless, at some point during this period, the potential for sudden onset of voice loss increases. In addition to the incremental build-up, any additional increase in cognitive anxiety, such as prior to and during a performance, could significantly increase the risk of sudden voice loss. Hence there would need to be expert judgment made to know the safe duration for any extended period of higher-pitched singing for the changing voice. Consequently, it is recommended that the practice of singing with an extended range during adolescence would need further research before it could be 
assumed to be based on a sound pedagogical judgment.

It has been shown that the attitude and effectiveness of the conductor are key elements for the effort, self-worth and success of boys' choir members [47, 48]; it would be assumed that a recognized choral foundation would be best placed to provide this experience for the children concerned. In the environment for this particular research, the background and training provided by the choral conductors for the boys tends to be primarily musical, without any specific reference to vocal technique. Many choral foundations also employ a singing teacher, in order to guide the boys in the healthiest voice use. However, singing teachers in the UK are not formally trained, licensed or regulated; specific knowledge of children's voices is generally gained through personal experience rather than formal training. Due to the paucity of formal training in vocal health and singing pedagogy, the level and consistency of training offered to choristers in UK cathedrals is at best, variable.

The evidence from vocal health research, Physical Stress Theory and Catastrophe Theory, and the information derived from detailed acoustic analysis of voice production suggest that the appearance of relatively easy phonation in the upper pitch range can be deceptive. There is a high level of expertise needed in order to finely judge the approaching tipping point into vocal catastrophe, and this may in fact be impossible to anticipate. The implications for educators are that the longitudinal development of singing habits will be enhanced for boys who move to singing with their new baritone range as their larynx grows, rather than remaining in a treble range. This will ensure a satisfactory transition on the way to discovering their young adult voice, whether they become a tenor, baritone, bass or even countertenor. In order to ensure a safe and fulfilling musical experience for all concerned, it is recommended that until further research in undertaken, that the Cooksey system is implemented to evaluate the changing voice rather than the extended range system.

\section{Appendices:}

Recordings of the sound files are available on the author's website: www.jenevorawilliams.com, Resources, Research Publications, Published Papers in Refereed Journals, Sound files for What Pitch Range is Best for Boys during Voice Change? 
The tests consist of the following range of voice activities (Table 1):

\begin{tabular}{|l|l|l|}
\hline & Activity & Purpose \\
\hline 1 & $\begin{array}{l}\text { Counting backwards from 20 to } 1 \\
\text { normal, loud, very loud and quiet }\end{array}$ & $\begin{array}{l}\text { This demonstrates comfortable, } \\
\text { customary fundamental frequency } \\
\text { (Fo) at varying degrees of } \\
\text { loudness, and the effect of } \\
\text { 'projecting' the speaking voice on } \\
\text { voice quality }\end{array}$ \\
\hline 2 & $\begin{array}{l}\text { Reading aloud the first paragraph } \\
\text { of the standard passage "Arthur } \\
\text { the Rat" (see Appendix) }\end{array}$ & $\begin{array}{l}\text { This text has a carefully distributed } \\
\text { selection of phonemes, enabling a } \\
\text { thorough perceptual evaluation of } \\
\text { voice quality }\end{array}$ \\
\hline 3 & $\begin{array}{l}\text { Singing a sustained single pitch; } \\
\text { beginning quietly, increasing and } \\
\text { then decreasing the loudness. On } \\
\text { both D4 and D5 }\end{array}$ & $\begin{array}{l}\text { This demonstrates technical vocal } \\
\text { control, maintaining stable pitch } \\
\text { with variable intensity }\end{array}$ \\
\hline 4 & $\begin{array}{l}\text { Singing a pitch glide starting on } \\
\text { G4; to slide up as far as possible, } \\
\text { down as far as possible and to } \\
\text { return to the starting note }\end{array}$ & $\begin{array}{l}\text { This demonstrates total pitch } \\
\text { range and alterations in voice } \\
\text { quality with pitch changes }\end{array}$ \\
\hline 5 & $\begin{array}{l}\text { Singing a two-octave major scale, } \\
\text { from G3, up and down }\end{array}$ & $\begin{array}{l}\text { This demonstrates changes in } \\
\text { voice quality over controlled pitch } \\
\text { changes and register transitions }\end{array}$ \\
\hline 6 & $\begin{array}{l}\text { Singing one verse of the hymn } \\
\text { unact, the very thought of thee" } \\
\text { unaccompanied (see Appendix) }\end{array}$ & $\begin{array}{l}\text { This melody has a relatively small } \\
\text { pitch range and a mainly stepwise } \\
\text { melodic contour }\end{array}$ \\
\hline
\end{tabular}

Table 1: Acoustic recording protocol for choristers 2003 - 5

\section{References}

1. Cooksey, J.M., The Development of a Contemporary, Eclectic Theory For The Training And Cultivation of The Junior High School Male Changing Voice: PART II SCIENTIFIC AND EMPIRICAL FINDINGS; SOME TENTATIVE SOLUTIONS. The Choral Journal, 1977. 18(3): p. 5-16.

2. Leck, H.H., On the Voice: the boy's expanding voice: take the high road. Choral Journal, 2009. 49(11): p. 49-60.

3. Phillips, K.H., Teaching kids to sing. 1996, Belmont, California: Schirmer.

4. $\quad$ Morris, R., et al., Phonational profiles of male trained singers and nonsingers. Journal of Voice, 1995. 9(2): p. 142-148.

5. Lloyd, R.S., et al., Chronological Age vs. Biological Maturation: Implications for Exercise Programming in Youth. Journal of Strength and Conditioning Research, 2014. 28(5): p. 1454-1464. 
6. Tanner, J.M., Growth at adolescence: with a general consideration of the effects of hereditary and environmental factors upon growth and maturation from birth to maturity. 1964, Oxford: Blackwell Scientific.

7. Harries, M.L., et al., Changes in the male voice during puberty: speaking and singing voice parameters. Logopedics Phoniatrics Vocology, 1996. 21: p. 95-100.

8. $\quad$ Fuchs, M., et al., Prediction of onset of voice mutation in singers of professional boys' choirs: investgation of members of the Thomanerchor, Leipzig. Folia Phoniatrica Logopedia, 1999. 51(6): p. 261-271.

9. Cooksey, J.M., Voice transformation in male adolescents, in Bodymind and Voice, L. Thurman and G. Welch, Editors. 2000, The VoiceCare Network: Minnesota, USA. p. 718-738.

10. Böhme, G. and G. Stuchlick, Voice profiles and standard voice profile of untrained children. Journal of Voice, 1995. 9(3): p. 304-307.

11. Sederholm, E., Prevalence of hoarseness in ten-year-old children. Scandinavian Journal of Logopedics and Phoniatrics, 1995. 20: p. 165173.

12. Williams, J., The implications of intensive singing training on the vocal health and development of boy choristers in an English Cathedral Choir, (unpublished doctoral dissertation), University of London: London. 2010

13. Cooper, I., Changing voices. Music Educators Journal, 1962. 48: p. 148-151.

14. Ashley, M., Contemporary Choral Work with Boys. 2014, Oxford: Compton Publishing Ltd.

15. Whiteside, S.P., C. Hodgson, and C. Tapster, Vocal characteristics in preadolescent and adolescent children: a longitudinal study. Logopedics Phoniatrics Vocology, 2002. 27: p. 12-20.

16. Marshall, W.A. and J.M. Tanner, Puberty, in Human growth: a comprehensive treatise, F. Falkner and J.M. Tanner, Editors. 1986, Plenum Press: London. p. 171-209.

17. Hollien, H., R. Green, and K. Massey, Longitudinal research on adolescent voice change in males. Journal of the Acoustical Society of America, 1994. 96: p. 2646-2654.

18. Soliman, A., M. Khadir, and M. Asfour, Testosterone treatment in adolescent boys with constitutional delay of growth and development. Metabolism, 1995. 44(8): p. 1013-1015.

19. Harries, M.L., et al., Changes in the male voice at puberty: vocal fold length and its relationship to the fundamental frequency of voice. The Journal of Laryngology and Otology, 1998. 112: p. 451-454.

20. Bailey, D.A., R.M. Malina, and R.L. Mirwald, Physical activity and growth, in Human Growth: a comprehensive treatise, F. Falkner and J.M. Tanner, Editors. 1986, Plenum Press: London. p. 147-170.

21. Malina, R.M., Growth of muscle tissue and muscle mass, in Human growth: a comprehensive treatise, F. Falkner and J.M. Tanner, Editors. 1986, Plenum Press: London. p. 77-99.

22. Scherer, K.R., et al., The expression of emotion in the singing voice: Acoustic patterns in vocal performance. The Journal of the Acoustical Society of America, 2017. 142: p. 1805-1815.

23. Kayes, G., How does genre shape the vocal behaviour of female singers?, (unpublished doctoral dissertation), University of London, 2014. 
24. Hazlett, D., O.M. Duffy, and S.A. Moorhead, Review of the Impact of Voice Training on the Vocal Quality of Professional Voice Users: Implications for Vocal Health and Recommendations for Further Research. Journal of Voice, 2011. 25(2): p. 181-191.

25. Mueller, M. and K. Maluf, Tissue Adaptation to Physical Stress: A Proposed "Physical Stress Theory" to Guide Physical Therapist Practice, Education, and Research. Physical Therapy, 2002. 82(4): p. 383-403.

26. Hardy, L. and J. Fazey. The Inverted-U Hypothesis: a catastrophe for sport psychology? in Annual Conference of the North American Society for the Psychology of Sport and Physical Activity. 1987. Vancouver.

27. Yerkes, R. and J. Dodson, The relation of strength of stimulus to rapidity of habit-formation. Journal of Comparative Neurology and Psychology. , 1908. 18(5): p. 459-482.

28. Papageorgi, I., A. Creech, and G.F. Welch, Perceived performance anxiety in advanced musicians specializing in different musical genres. Psychology of Music, 2011.

29. Mackinnon, S.E. and C.B. Novak, Pathogenesis of cumulative trauma disorder. Journal of Hand Surgery, 1994. 19(5): p. 873-883.

30. Roy, N., Functional Dysphonia. Current Opinion in Otolaryngology \& Head and Neck Surgery, 2003. 11(3): p. 144-148.

31. Howard, D., G.A. Lindsey, and B. Allen, Towards the quantification of vocal efficiency. Journal of Voice, 1990. 4(3): p. 205-212.

32. Barlow, C.A. and D. Howard, Electrolarygographically derived voice source changes of child and adolescent singers. Logopedics Phoniatrics Vocology, 2005. 30(3-4): p. 147-157.

33. http://www.speech.kth.se/wavesurfer/.

34. http://www.phon.ucl.ac.uk/resource/sfs/wasp.htm.

35.

http://www.softwarecasa.com/snagit.html?gclid=CObzoKOboZ4C FUYA4wodBW-hlA.

36. Gauffin, J. and J. Sundberg, Spectral correlates of glottal voice source waveform characteristics. Journal of Speech and Hearing Research, 1989. 32: p. 556-565.

37. Howard, D.M., Electroglottography/electrolaryngography, in The Larynx, M.P. Fried and A. Ferlito, Editors. 2009, Plural Press: San Diego. p. 227243.

38. Welch, G. and P.J. White, The developing voice: educational and vocal efficiency - a physical perspective. Bulletin of the Council for Research in Music Education, 1993. 119: p. 146-156.

39. Abberton, E., D. Howard, and A. Fourcin, Laryngographic assessment of normal voice: a tutorial. Clinical Linguistics and Phonetics, 1989. 3(3): p. 281-296.

40. Jilek, C., J. Marienhagen, and T. Hacki, Vocal stability in functional dysphonic versus healthy voices at different times of voice loading. Journal of Voice, 2004. 18(4): p. 443-454.

41. Group, B.M.E.R., Mapping music education research in the UK. Psychology of Music, 2004. 32(3): p. 239-290. 
42. Howard, D., Variation of electrolaryngraphically derived closed quotient for trained and untrained adult singers. Journal of Voice, 1995. 9(2): p. 163172.

43. Reid, K., et al., The acoustic characteristics of professional opera singers performing in chorus versus solo mode. Journal of Voice, 2007. 21(1): p. 35-45:

44. Howard, D. and D. Murphy, Voice Science, Acoustics, and Recording. 2008, San Diego: Plural Publishing.

45. Howard, D. and J. Williams. An investigation of 'ring' in the voices of highly trained child singers. in PEVOC8. 2009. Dresden.

46. Howard, D., Electroglottography/electrolaryngography, in The Larynx, M.P. Fried, Editor. 2009, Plural: Abingdon, Oxon.

47. Daugherty, J. and D. Hedden, Audition procedures for community-based children's choirs. Bulletin of the Council for Research in Music Education, 2006. 167: p. 33-45.

48. Durrant, C, Making choral rehearsal seductive: implication for practice and choral education. Research Studies in Music Education, 2000. 15: p. 40-49. 